\title{
Structure Design of Automobile Engine Mounting System
}

\author{
Zhao Tianpeng $^{\mathrm{a}}$ \\ Beijing Research Centre of Sustainable Energy and Building \\ Beijing University of Civil Engineering and Architecture \\ Beijing 100044, China \\ a13811913654@163.com
}

\author{
Liu Yongfeng ${ }^{\mathrm{b}}$, Shi Yan ${ }^{\mathrm{c}}$, Wang $\mathrm{Na}^{\mathrm{d}}$ \\ Beijing Research Centre of Sustainable Energy and Building \\ Beijing University of Civil Engineering and Architecture \\ Beijing 100044, China \\ bliuyongfeng@bucea.edu.cn, csyst1988@163.com, \\ d13811920259@163.com
}

\begin{abstract}
In order to understand the principle of the engine mounting system and design the suspension element, the theory which is useful in the design of Engine Mounting System is introduced. And the problems that ought to be taken noticed in the Engine Mounting System design is carried out. A lot of calculating formulas about vibration are derived to deepen the understanding of principle. Then use CATIA to establish 3D model of the vehicle engine mount.
\end{abstract}

Keywords—engine mounting; vibration; 3D model

\section{INTRODUCTION}

When the engine is moving, it will produce a reciprocating, unbalanced vibration, and it is very strong. The vibration of the engine will pass through the chassis and the body into the car, and then pass to the driver and passengers. It is easy to make them produce fatigue and may cause a lot of unnecessary harm. First, the life of the vehicle is shortened due to the fatigue damage of the frame and auto parts. Second, it will aggravate the noise level of the car. The driver will have a sense of fatigue due to vibration and noise. It is easy to bring traffic safety problems. The vibration will let passengers discomfort, Affect people's mood to travel. In addition, uneven road surface will produce random excitation and it will pass through the wheel and the frame to the engine. The vibration will affect the normal work of the engine, and then reduce the engine's reliability and stability, and even make direct damage to the engine.

In 1979, Johnson used system's natural frequency and the vibration coupling as the objective function to optimize the positions and the stiffness of the suspension. The results showed that the vibration coupling between different degrees of freedom in the system is greatly reduced, and it ensures that the six order natural frequency of the system is within the desired range. And he became the first scientist in the design of the suspension system.

In 1992,Shangguan Wenbin et al made a large number of experiments and investigation to the market,. They summed up the problems that may be encountered in the optimization and the general method of the optimization in the design of engine mounting system. In their experiments, they made the natural frequency of the system as objective function, and used the system decoupling method and impact center theory to establish optimization model and made the optimization calculation. Finally good results have been obtained. They has made a great contribution to engine suspension system development.

In 1994, Wang Ligong et al made a comprehensive discuss in the domestic development of the suspension structure of the suspension for the first time in china. The structure, working principle, characteristics and development trend of typical hydraulic suspension structures are discussed.

In 1999,Qiu Xin et al according to the previous summary and combining with the situation of the development of the car they optimized and analyzed the engine suspension system. They compare the traditional rubber suspension and the emerging hydraulic suspension in the vibration isolation characteristics and then analyze it. The results are verified by the simulation experiments, and they make a great contribution to the development of the suspension system.

\section{WORKING PRINCIPLE AND MODEL OF ENGINE MOUNTING SYSTEM}

Simplify the suspension system of the automobile engine to the simple mechanical model shown in Figure 1.

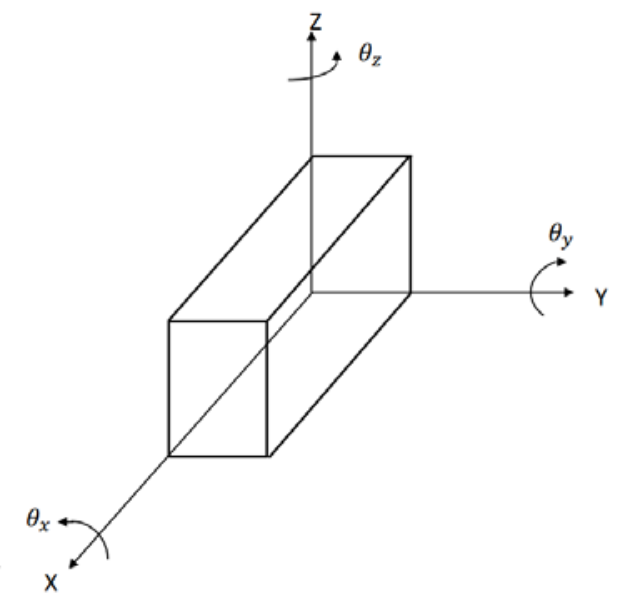

Fig. 1. Suspension system model 
The natural frequency of the vehicle engine and its suspension is about $6 \sim 30 \mathrm{~Hz}$, and the system can be seen as a rigid body motion in the frequency range. So it can be simplified into a cube. Build space coordinate system. The points are represented by $\mathrm{X}, \mathrm{Y}, \mathrm{Z}$. Using $\theta_{x}, \theta_{y}, \theta_{z}$ to represent the three corners of the axis parallel to the fixed axis. So (1) can be used to express the engine's six degrees of freedom vector.

$$
Q=\left\{\text { x y z } \theta_{\mathrm{x}} \theta_{y} \theta_{\mathrm{z}}\right\}^{\mathrm{T}}
$$

The engine assembly is considered as a rigid body motion, and its kinetic energy is(2).

$$
\begin{aligned}
T & =\frac{1}{2}\left(m \dot{x}^{2}+m \dot{y}^{2}+m \dot{z}^{2}+J_{x} \dot{\theta}_{x}{ }^{2}+J_{y} \dot{\theta}_{y}{ }^{2}+J_{z} \dot{\theta}_{z}^{2}\right) \\
& -J_{x y} \dot{\theta}_{x} \dot{\theta}_{y}-J_{y z} \dot{\theta}_{y} \dot{\theta}_{z}-J_{x z} \dot{\theta}_{x} \dot{\theta}_{z}
\end{aligned}
$$

Transform (2) into matrix, as in (3)

$$
T=\frac{1}{2} \dot{Q}^{T} M \dot{Q}
$$

In (3), $Q=\left\{\mathrm{x} \mathrm{y} \mathrm{z} \theta_{\mathrm{x}} \theta_{y} \theta_{\mathrm{z}}\right\}^{\mathrm{T}}$ is a generalized velocity column, $\mathrm{M}$ is the mass matrix.

$$
M=\left[\begin{array}{cccccc}
m & 0 & 0 & 0 & 0 & 0 \\
0 & m & 0 & 0 & 0 & 0 \\
0 & 0 & m & 0 & 0 & 0 \\
0 & 0 & 0 & J_{x} & -J_{x y} & -J_{z x} \\
0 & 0 & 0 & -J_{x y} & J_{y} & -J_{y z} \\
0 & 0 & 0 & -J_{z x} & -J_{y z} & J_{z}
\end{array}\right]
$$

In (4), $\mathrm{m}$ is the quality of the engine, $J_{x} 、 J_{y} 、 J_{z}$ is the engine power assembly of the $\mathrm{X}, \mathrm{Y}, \mathrm{Z}$ axis of inertia, $J_{x y} 、 J_{x z} 、 J_{y z}$ is the inertial product of the engine plane.

$$
\text { Let } J_{x y}=J_{x z}=J_{y z}=0 \text {, we can get (5). }
$$$$
T=\frac{1}{2}\left(m \dot{x}^{2}+m \dot{y}^{2}+m \dot{z}^{2}+J_{x} \dot{\theta}_{x}^{2}+J_{y} \dot{\theta}_{y}^{2}+J_{z} \dot{\theta}_{z}^{2}\right)
$$

We can assume that the number of the support element is n, and the characteristics of each supporting element are not same. Use $\left(x_{i}, y_{i}, z_{i}\right)$ to represent the support element. Use (6) to represents the potential energy of the system.

$$
U=\frac{1}{2} \sum_{i=1}^{n}\left(k_{u i} \Delta u_{i}^{2}+k_{v i} \Delta v_{i}^{2}+k_{s i} \Delta s_{i}^{2}\right)
$$

In (6), $\mathrm{K}$ represents the main rigidity, $\Delta u_{i}, \Delta v_{i}, \Delta s_{i}$ represents the shaft deformation.

Transform (6) into matrix, as in (7).

$U=\frac{1}{2} \sum_{i=1}^{n}\left\{\begin{array}{lll}\Delta u_{i} & \Delta v_{i} & \Delta s_{i}\end{array}\right\}\left[\begin{array}{ccc}k_{u i} & 0 & 0 \\ 0 & k_{v i} & 0 \\ 0 & 0 & k_{s i}\end{array}\right]\left\{\begin{array}{l}\Delta u_{i} \\ \Delta v_{i} \\ \Delta s_{i}\end{array}\right\}=\frac{1}{2} \sum_{i=1}^{n} \Delta W_{i}^{T} D_{i} \Delta W_{i}$

In (7)

$$
\Delta W=\left\{\begin{array}{l}
\Delta u_{i} \\
\Delta v_{i} \\
\Delta s_{i}
\end{array}\right\}, D_{i}=\left[\begin{array}{lll}
k_{u i} & 0 & 0 \\
0 & k_{v i} & 0 \\
0 & 0 & k_{s i}
\end{array}\right]
$$

Use (9) to change (8), and get (10).

$$
\Delta W=C_{i} \Delta X_{i}
$$

In (9), $C=C_{s i} \cdot C_{u i} \cdot C_{v i}$, and $C_{u i}=\left[\begin{array}{ccc}1 & 0 & 0 \\ 0 & \cos \theta_{u i} & \sin \theta_{u i} \\ 0 & -\sin \theta_{u i} & \cos \theta_{u i}\end{array}\right]$

$C_{v i}=\left[\begin{array}{ccc}\cos \theta_{v i} & 0 & -\sin \theta_{v i} \\ 0 & \cos \theta_{v i} & 0 \\ \sin \theta_{v i} & 0 & \cos \theta_{v i}\end{array}\right] C_{s i}=\left[\begin{array}{ccc}\cos \theta_{s i} & \sin \theta_{s i} & 0 \\ -\sin \theta_{s i} & \cos \theta_{s i} & 0 \\ 0 & 0 & 1\end{array}\right]$

$$
U=\frac{1}{2} \sum_{i=1}^{n} \Delta X_{i}^{T} C_{i}^{T} D_{i} C_{i} \Delta X_{i}
$$

When the system is in small displacement, (11) can be used to express relationship between the displacement and deformation of the system.

$$
\Delta X_{i}=T_{i} Q
$$

In (11),

$$
T_{i}=\left[\begin{array}{cccccc}
1 & 0 & 0 & 0 & z_{i} & -y_{i} \\
0 & 1 & 0 & -z_{i} & 0 & x_{i} \\
0 & 0 & 1 & y_{i} & -x_{i} & 0
\end{array}\right]
$$

Take (11) into (10), get (12) .

$$
U=\frac{1}{2} \sum_{i=1}^{n} Q^{T} T_{i}^{T} C_{i}^{T} D_{i} C_{i} T_{i} Q=\frac{1}{2} Q^{T}\left(\sum_{i=1}^{n} T_{i}^{T} C_{i}^{T} D_{i} C_{i} T_{i}\right) Q=\frac{1}{2} Q^{T} K Q
$$

Use (12),the stiffness matrix of the system can be got.

$$
K=\sum_{i=1}^{n} T_{i}^{T} C_{i}^{T} D_{i} C_{i} T_{i}
$$

\section{ENGINE MOUNTING SYSTEM DESIGN}

\section{A. Arrangement method of engine mount elastic support}

If the form of suspension is no regularity, it may lead to the phenomenon of coupling of multiple directions of mounting 
elements. Therefore, in the actual application and manufacturing process, the engine mount system must in accordance with a certain rule. I choose to use flat type. This kind of arrangement is often used, and it is a kind of traditional layout. It's easy to install, and the layout is simple.

\section{B. Number and position of the supporting point}

The engine mounting system usually uses 3 point support or 4 point support. Make reasonable distribution of the load according to the exciting force. No matter how the vehicle vibrates, the suspension system can always let the supporting points in the same plane. This is the advantage of three points. This advantage can greatly improve the body's stress. I choose to use 3 point support.

\section{Design of suspension element}

The engine's Gas pressure distribution is not uniform at idle speed and it is easy to produce low frequency and high amplitude vibration. The uneven pavement can also produce this vibration. Therefore, the suspension element should have low frequency, large damping and high stiffness characteristics. On the other side, to reduce the noise in the car and improve the stability of the operation, the suspension element should have high frequency, small damping and low stiffness characteristics.

The model of the suspension element is established by CATIA. The research and of CATIA is a flagship software development by the Dassault company. As a very important part of PLM, it can help companies design their products, and support the design, analysis, simulation, assembly and maintenance of all industrial design process from the pre project phase. Figure 2- Figure 6 are the model of the main components. Figure 7 is the assembly diagram.

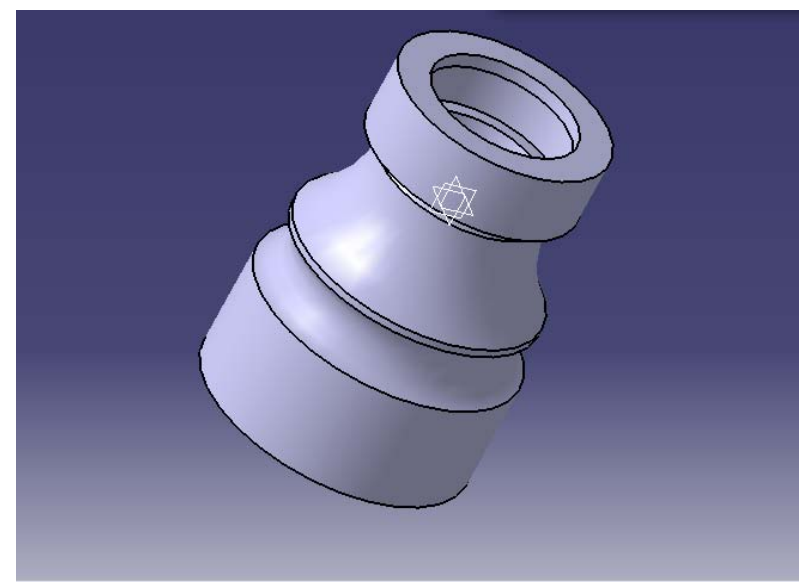

Fig. 2. Rubber spring

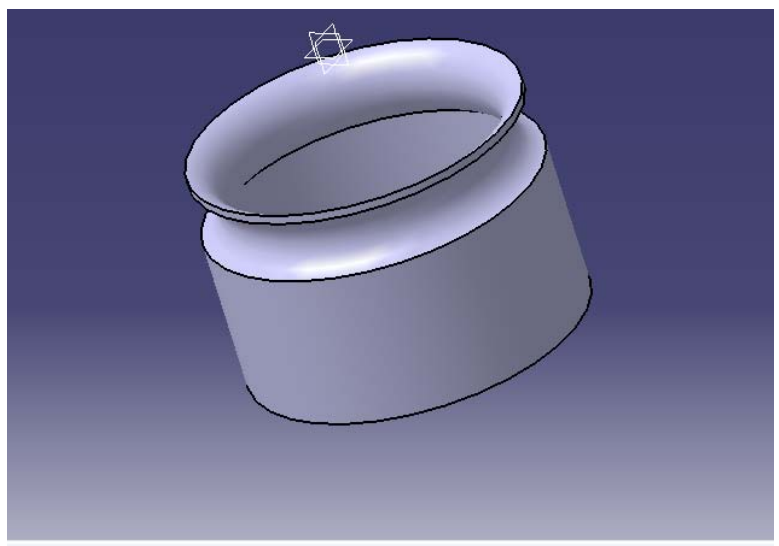

Fig. 3. Outer steel sleeve

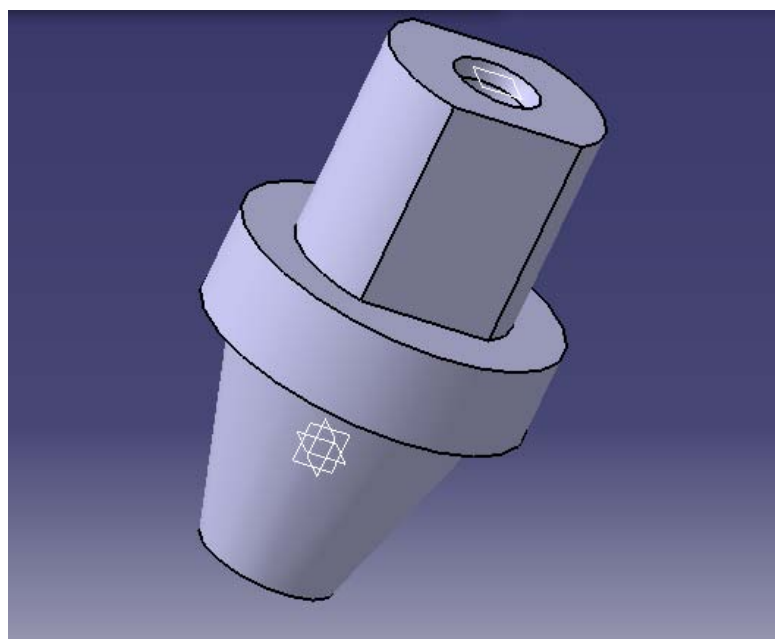

Fig. 4. The inner core

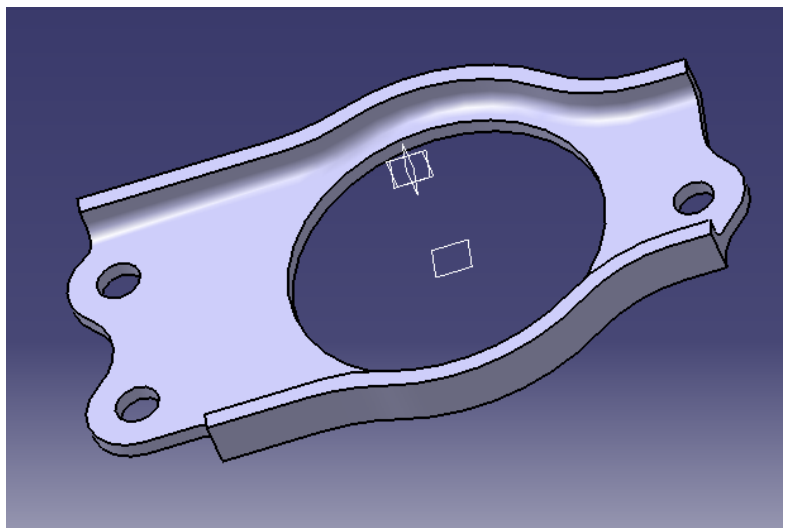

Fig. 5. Bracket 


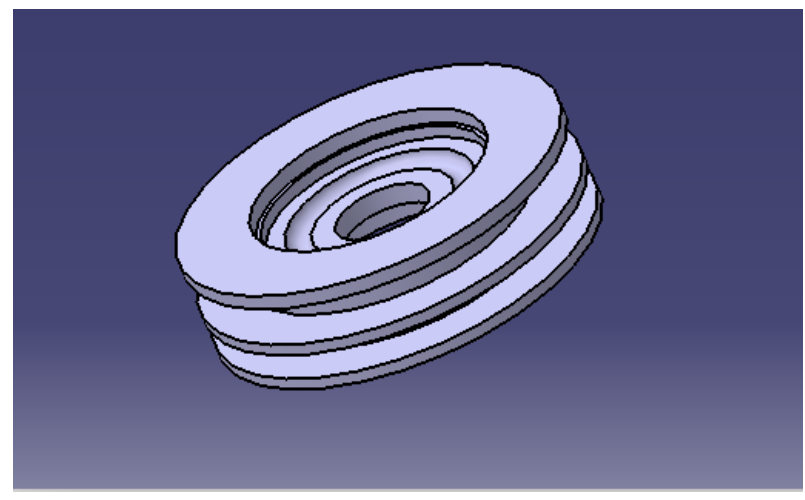

Fig. 6. Runner plate

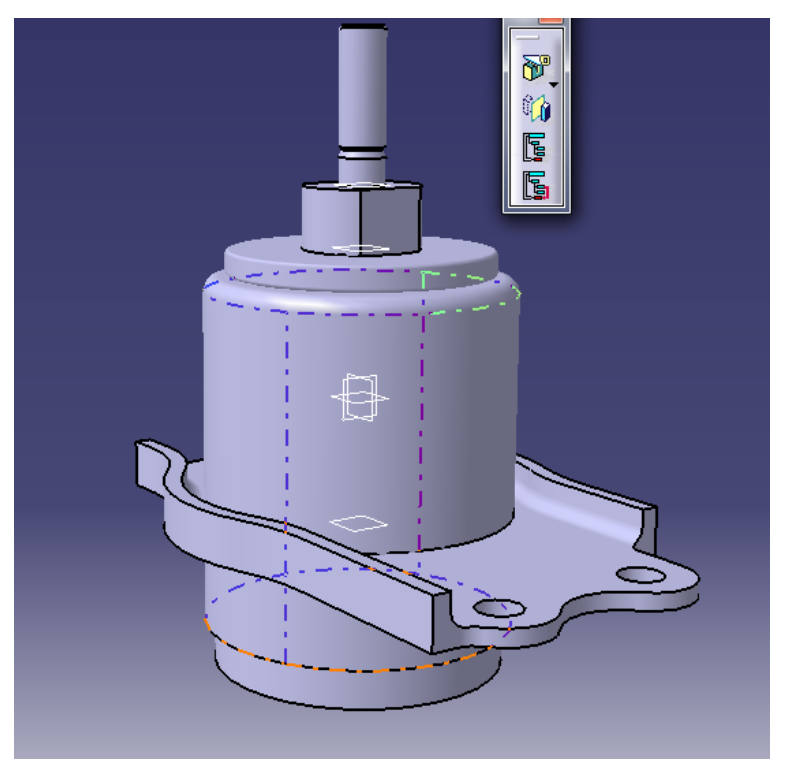

Fig. 7. Assembly diagram

\section{CONCLUSIONS}

A simple analysis and description of the vibration of the vehicle is carried out. A lot of calculating formulas are derived. Using the formula analysis to understand the principle of the engine mount, and determine the vehicle engine mount design ideas. The 3D model of the vehicle engine mount is set up by using CATIA. And it carry out a new type of the suspension element.

\section{ACKNOWLEDGMENT}

The study was sponsored by The Importation and Development of High-Caliber Talents Project of Beijing Municipal Institutions (CIT\&TCD20140311) and Beijing Natural Science Fund (SQKZ201510016004).

\section{REFERENCES}

[1] Johnson Stephen . Computer Optimization of Engine Mounting Systems. SAE paper 790974

[2] Geck PE , Patton RD . Front Wheel Drive Engine Mount Optimization. SAE paper 840736

[3] Yunhe Yu.Naganathan Nagi G.A Literature Review of Automotive Vehicle Engine Mounting Systems.Mechanism and Machine Theory.2001,36(1):123 142

[4] MJC Supot Hannongbua. Modification of front wheel drive vehicle engine mounting system for reduction of drive-away shudder. Icsv 2013

[5] Francesco Sorge. Analysis of the Ratio Shife Transient in V-Belt Variators[C]. Proceedings of the 11th World Congress in Mechanism and MachineScience, Tianjin, China, April 1-4, 2004:858-862. 